\title{
Discussion on Teaching of EDA in Communication Engineering
}

\author{
Duo Peng ${ }^{1, a}$ and Hao Chen ${ }^{1, b}$ \\ ${ }^{1}$ School of Computer and Communication, Lanzhou University of Technology \\ Lanzhou 730050, China \\ apengduo7642@163.com, ${ }^{\mathrm{b}}$ chenhao@lut.cn
}

Keywords: EDA technology; Teaching; Communication Engineering; Experiment.

\begin{abstract}
EDA technology has been widely used and has an important role in the field of electronic information and communication engineering. Combined with teaching practice, illustrates the importance of EDA teaching in communication engineering students. We explored the requirements of the course in terms of choice of teaching content, classroom teaching and practical aspects of teaching. Training objectives for communication engineering major proposed EDA teaching should pay attention to several issues, in order to achieve the goal of training and cultivating students' innovative spirit and practical ability. Further improve the level of professional development and teaching quality.
\end{abstract}

\section{Introduction}

EDA called "Electronic Design Automation", EDA technology is developed rapidly in the 1990s, and is the latest research results integrated modern electronic technology and computer technology, is a technology of engaged in electronic circuit design and analysis, mainly includes three aspects: electronic circuit design, computer simulation and circuit analysis, printed circuit board design automation.

The use of EDA design tools, people can make electronic circuit simulation, electronic systems or integrated circuit chip design and testing. This greatly reduces the design time significantly improves the performance and reliability of electronic circuits and systems. Enables designers freed from the heavy repetitive work, and to focus on higher level of design work of the electronic system modeling, algorithms and behavioral descriptions. That a large number of specific low-level works by EDA tools and computers do it. It can be say, in today's age, there is no development and application of EDA technology, people will not be able to effectively design and development of modern electronic circuits or electronic systems, there would be no modern information society of today [1-3].

\section{Necessity of Communication Engineering Conducted EDA Education}

EDA technology is a major technology in the 21 st century electronic design. If you do not use the EDA approach to design of modern electronic systems becomes moot to talk about. Such as large aerospace electronic systems, a variety of communication network systems, computer network systems, etc. If you leave the EDA will not be completed. In the modern electronic information enterprises, regardless of the size of their business, from product design to production processes are inseparable from the EDA technology, so that EDA technology is the must master the technique of modern electronic engineer.

According to professional training plan of communication engineering, students receive basic training in communication engineering practice teaching, and have a basic ability in modern communication systems and network design, development, commissioning and engineering applications. To grasp the analysis and design methods of communication systems and communication networks, with the basic ability to design, development, debugging and testing, application communication systems and communication networks. In many areas of the actual teaching are related to the EDA technologies, such as "Computer Communications" course design, 
"Communication Circuits" curriculum design, integrated communications training, graduation design. But the lack of an independent practice teaching, causing students to feel all learned a little, but did not learn the fine, feeling useful, but little knowledge of the situation. Especially in graduate design students is even more evident in the weak capacity in this regard [4,5].

Therefore, it is necessary to set up an independent practice teaching focused on teaching EDA technology, master the basic design methodologies, skilled use of one or two popular EDA design software. Enable students to lay a more solid foundation, with a strong ability to improve the quality of teaching in college courses.

\section{EDA Teaching and Students' Engineering Practice}

Engineering practice is based on the characteristics of the culture of engineering students, in particular the practice environment for students in electronics, information systems, engineering and technical training conducted. It has the following meanings:

First, through practical training, students can develop the ability of engineering practice, so that students from the "merely have known" to "can still make a difference", so as to promote efficient conversion of knowledge to the students abilities; more It is important also to develop students ability to innovate, inspire students' creative desires.

Second, the students through hands-on, making direct contact with perceptual knowledge, to reinforce its image of thinking, to achieve common development of abstract thinking and thinking in images;

Third is the "creative production", that is, from the students themselves creative design of electronic circuits, electronic small products and self-produced, students apply knowledge to solve practical problems and innovative ability. When building and implementing experimental teaching, to follow the law of human cognition, full consideration of individual differences of students and students from low-grade to high-grade the different stages of growth. Experimental Teaching gradual process to follow to make that: basic skills training $\rightarrow$ system design $\rightarrow$ Ability to work independently Ability. Students of the lower grades, mainly through basic experimental courses, course choose to do experiments to develop their basic skills [6,7] .

Once students have the basic skills, it is necessary to attention to develop their the ability of the system design, which should be achieved through curriculum design, electronic system design experiments. Ability to work independently to achieve mainly through independent development issues involved in scientific research and other aspects of instructors. Basic skills, integrated design and innovative design that the proportion of three parts shown in Figure 1.
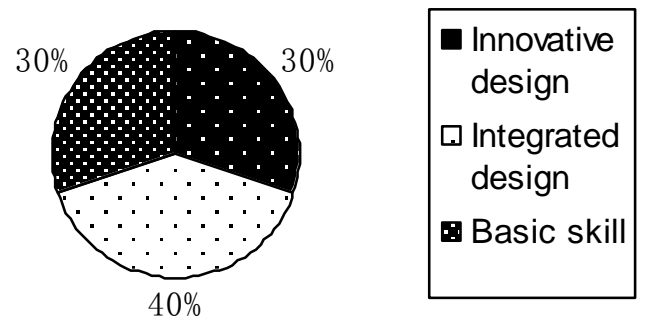

Figure. 1 Teaching content proportion of three parts.

\section{Teaching Content and Requirements of EDA Technology in Communication Engineering}

Because of EDA technology covers a wide range, rich in content, from teaching and practical point of view, to improve the teaching of EDA technology in communication engineering, we should first understand and determine the EDA should have teaching content and requirements. According to the 
training objectives and related professional teaching status of other universities EDA research and analysis, that the conduct of EDA technology teaching should include the following:

Communication Electronic Circuit Design CAD. That education students to master communication electronic circuit CAD technology, enables schematic entry and design, PCB layout and verification on the computer. So that students can be more fluent in communication electronic circuit CAD design.

System Design and Simulation. In the process of modern communications, electronics design and application, system design and simulation analysis is time-saving, cost-saving design, improve design efficiency as an essential part. Therefore, to carry out teaching in university education and the promotion of EDA system design and simulation analysis techniques should become a focus of communication and information content class professional education, conduct teaching system design and simulation analysis, mainly using EDA software development tools to be carried

Currently, there are several good performance circuit and system analysis, design and simulation tools, which provide a large number of components, tools, libraries, and a variety of on-screen virtual test instruments, can be a variety of digital-analog hybrid simulation and circuit analysis. Once the circuit or system designs, simulation ended, you can quickly auto-routing based on PCB layout design results, and produce practical PCB. For example, the well-known Protel series circuits design software, Multisim simulation design software, EWB (Electronics Workbench) software. These powerful simulation design software, work intuitive interface with a variety of devices and instruments library, support circuit devices and test equipment screen graphics calls and analog / digital circuits hybrid simulation. In the international electronic design and education generally favored by the people. But the price of these software development systems is more expensive, require more capital investment in order to establish a teaching platform [8,9]. At present, in Communication Engineering Teaching (Author's school), using the ratio of three software shown in Figure 2.

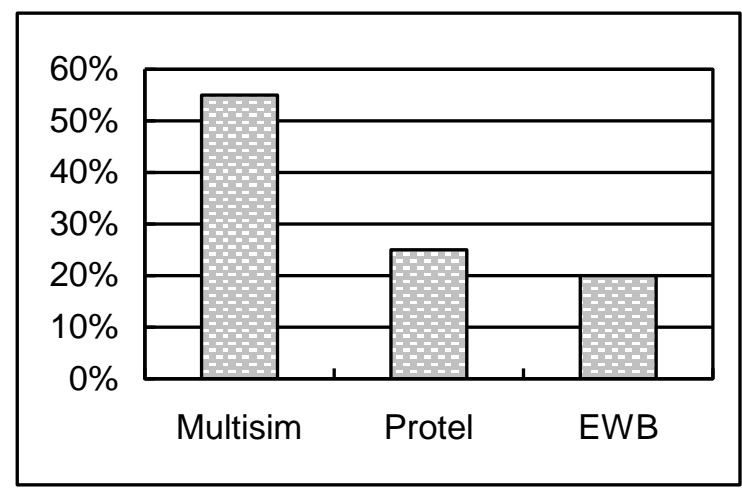

Figure. 2 Using the proportion of three software

\section{Communication Engineering Teaching EDA Technical Issues should be Noted}

EDA technology is developing very fast, as a teaching should be based on EDA technology for students to master basic methods and applications, understand EDA design ideas and preliminary study using the typical method of EDA design tools, students need to continue to work in the future learning, so they learn to master the advanced technological capabilities culture is more important. EDA teaching teachers should engage in continuous learning to improve by themselves, the world's most advanced technology and the latest knowledge to their students, play a valuable role.

EDA teaching should follow the principle succinctly and more practice, that thirty percent of teaching, seventy percent practice, the use of multimedia teaching methods and situational approach. Classroom teaching based on difficulty speaking, about the application, speak equipment in use precautions. In experiments on board to acquire knowledge, application-oriented, try to organize content into a comprehensive, design issues, and by strengthening the usual operating assessment allows students to enhance learning initiative to change the passive situation of learning [10] . 
Graduation project is a very important part of the undergraduate teaching. Currently graduating class design engineering subject a lot, but due to less funding, device, inadequate equipment and other reasons, the result is often principle discussion and analysis. Not the actual debugging and verification, the student remains on the principle analysis, not through actual practice research design. Graduate design effect is not ideal. Therefore, it can be used in the process of graduation design, teaching students to design their own circuit simulation, presentation in his reply and ask students to make a prototype, prototype printed circuit boards must be drawn with Protel software, it will ensure graduation design quality, but also improve the students' practical ability and development capabilities, yet is a way to overcome the problem of graduate design now.

\section{Conclusion}

EDA technology is a revolution in the field of electronic design, it has penetrated into all areas of electronic information. For the student of communication majors master EDA technology can be broaden field of knowledge, and enhance market competitiveness. Therefore, in communication engineering specialty EDA is an important means of teaching students' quality education and innovative ability and will further improve the professional level of the building and teaching quality.

\section{Acknowledgments}

Thanks to Gansu Province Natural Science Foundation funding for this article. (No: 1308RJZA104)

\section{References}

[1] Zhu Na, Zhang Jingbao, EDA technology Practical Guide, Machinery Industry Press, Bei Jing, 2012.

[2] Wang Guoqiang, EDA Technology and Application, fourth ed.,Electronic Industry Press, Bei Jing,2013

[3] Li Yu EDA technology Teaching , J. Lanzhou University of Technology, 2006,32 (Teaching and Research Album): 183-184.

[4] Wu Wenliang. Promoting the Reform of Personnel Training, Focus on Improving the Ability of Innovative Practice [J] China Higher Education, 2008(11):1-3.

[5] Schank E C. Revolutionizing the Traditional Classroom Course, J. Communications of the ACM, 2001, 44 (12):21 - 24.

[6] Zeng Yongwei, LUI Guofan. Exploration and Design of Practice Teaching System Based on Engineering College, J. China Higher Education, 2009(9):53-54.

[7] Yang Shuzi. For the project, building the foundation for comprehensive development, J. China University teaching, 2006, 7:4-9.

[8] Biggs J. (Ed.) Teaching for Quality Learning at University, J. Open University Press, 2003.

[9] The Task Force on General Education, Harvard University, 2007.Report of the Task Force on

General Education. http: Nwww.harvard.edu.

[10] Jiao Su Ming, EDA Applications, second ed., Tsinghua University Press, Bei Jing, 2011. 